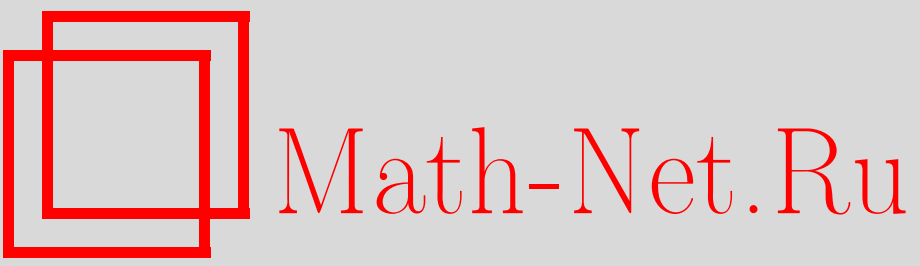

В. Р. Кудашев, Б. И. Сулейманов, Малоамплитудные дисперсионные колебания на фоне приближения нелинейной геометрической оптики, ТМФ, 1999, том 118, номер 3, 413-422

DOI: https://doi.org/10.4213/tmf714

Использование Общероссийского математического портала Math-Net.Ru подразумевает, что вы прочитали и согласны с пользовательским соглашением

http://www. mathnet.ru/rus/agreement

Параметры загрузки :

IP: 34.239 .49 .27

26 апреля 2023 г., 04:53:51 
ТЕОРЕТИЧЕСКАЯ

И МАТЕМАТИЧЕСКАЯ

ФИЗИКА

Toм 118, № 3

март, 1999

(C) 1999 г.

В.Р. Кудашев* , Б. И. Сулейманов*

\section{МАЛОАМПЛИТУДНЫЕ ДИСПЕРСИОННЫЕ КОЛЕБАНИЯ НА ФОНЕ ПРИБЛИЖЕНИЯ НЕЛИНЕЙНОЙ ГЕОМЕТРИЧЕСКОЙ ОПТИКИ}

Рассматривается асимптотика при $x^{2}+t^{2} \rightarrow \infty$ аналогов интеграла Пирси, описывающих влияние малой дисперсии на начало процессов самопроизвольного падения амплитуды приближения нелинейной геометрической оптики к решениям уравнений типа фокусирующего нелинейного уравнения Шредингера. Установлено, что при $x^{2}+t^{2} \rightarrow \infty$ для рассматриваемых специальных функций имеется область малоамплитудных двухфазовых высокочастотных колебаний, происходящих на фоне приближения нелинейной геометрической оптики ненулевой амплитуды.

1. В этой работе рассматривается асимптотика при $x^{2}+t^{2} \rightarrow \infty$ найденных в статье [1] совместных решений нелинейного уравнения Шредингера (НУШ)

$$
i P_{t}+P_{x x}+2|P|^{2} P=0
$$

и обыкновенного дифференциального уравнения (ОДУ)

$$
P_{x x x}+6|P|^{2} P_{x}-\frac{4 t P_{x}-2 i x P}{\beta}=0 .
$$

Исследуемые решения $P(t, x)$ являются аналогами интеграла Пирси $(\beta<0)$

$$
\int_{-\infty}^{\infty} \exp \left(-2 i\left(x \lambda+2 t \lambda^{2}+\beta \lambda^{4}\right)\right) d \lambda
$$

удовлетворяющего линейной части уравнений (1), (2).

Известный нелинейный аналог интеграла Пирси, исследовавшийся в [2], наряду с НУШ (1) также является [3] точным решением ОДУ (2). Однако в отличие от случая работ $[2,3]$, для решений из статьи [1] при больших значениях аргументов нелинейность доминирует над дисперсией.

${ }^{*}$ Институт математики Уфимского научного центра РАН, Уфа, Россия. E-mail: kudashev@imat.rb.ru; bis@imat.rb.ru 
Поясним только что сказанное. Подстановка $P=b \exp (i S)$ в уравнение $(2)$ дает следуюшую систему ОДУ на амплитуду $b$ и производную фазы $a=S_{x}$ :

$$
\begin{array}{r}
2 b_{x x} b-\left(b_{x}\right)^{2}=3 b^{2}\left(a^{2}-b^{2}\right)+\frac{4 t b^{2}}{\beta}+2 \gamma \\
a_{x x} b+3 a_{x} b_{x}+3 a b_{x x}=b\left(a^{3}+\frac{4 t a}{\beta}-6 a b^{2}-\frac{2 x}{\beta}\right) .
\end{array}
$$

Бездисперсионный предел (4) дает приближение [1]

$$
b_{0}^{2}=a_{0}^{2}+\frac{4 t}{3 \beta}, \quad \frac{5 \beta a_{0}^{3}}{2}+2 t a_{0}+x=0,
$$

являющееся главным членом асимптотики при $x^{2}+t^{2} \rightarrow \infty$ рассматриваемых решений (4) вне области, ограниченной ветвями параболы

$$
|x|=s_{0} t^{\frac{3}{2}}
$$

где $s_{0}=\sqrt{64 /(-27 \beta)}$. Внутри же этой области исследуемые решения должны стремиться к нулю.

2. Кривые (6) в точности совпадают со “следом каустики" для интеграла Пирси (3). А именно, при $s=s_{0}\left(s=x /|t|^{\frac{3}{2}}\right)$ уравнение

$$
s+4 f+4 \beta f^{3}=0
$$

решения которого определяют асимптотику (3) при $t \gg 1$, имеет кратные корни $f=f_{0}$. Асимптотика аналога интеграла (3) из [2] в окрестностях этих кривых описывается [3] в терминах решения второго уравнения Пенлеве

$$
v_{z z}+2 v^{3}=z v
$$

зависящего от переменной $z=-f_{0} \frac{1}{3} y=-f_{0} \frac{1}{3}\left(s-s_{0}\right) t^{\frac{4}{3}}$, и имеет вид $(c, d-$ вещественные, а $r$ - комплексная постоянные)

$$
\begin{aligned}
P= & t^{-\frac{1}{6}} \exp \left(-\frac{2 i t^{2}}{3 \beta}-2 i t^{\frac{2}{3}} f_{0} y+i c \ln t\right) q(y)+ \\
& +t^{-\frac{1}{2}} \exp \left(\frac{16 i t^{2}}{3 \beta}+4 i t^{\frac{2}{3}} f_{0} y-i d \ln t\right) r+\cdots
\end{aligned}
$$

где $q(y)$ - экспоненциально убываюшее при $y \beta f_{0} \rightarrow \infty$ решение ОДУ

$$
q_{y y}+2|q|^{2} q=\frac{y q}{3 \beta f_{0}}
$$

Приведенные в работе [3] рассуждения и смысл рассматриваемых в данной работе совместных решений уравнений (1) и (2) позволяют заключить, что и для них асимптотики в окрестности кривых (6) имеют вид (9). Только в данном случае главные члены 
асимптотик $q(y)$ при $y \beta f_{0} \rightarrow \infty$ определяются согласно (5) из бездисперсионного предела (10):

$$
2|q|^{2} q=\frac{y q}{3 \beta f_{0}} .
$$

Аналогичное свойство выполнено и при $y \beta f_{0} \rightarrow-\infty$ (такие функции $q(y)$ существуют [4]).

3. Оказывается, что при отрицательных $t$ для рассматриваемых здесь аналогов интеграла Пирси из статьи [1] имеются еше две выделенные кривые

$$
s= \pm \frac{14}{3} \sqrt{-\frac{16}{15 \beta}}
$$

Вдоль них асимптотику при $t \rightarrow-\infty$ также приходится задавать при помоши вещественных решений второго уравнения Пенлеве. Однако в отличие от кривых (6) на них приближение бездисперсионного предела остается гладким и главные члены исследуемых совместных решений (1) и (2) в ходе перехода через эти кривые по-прежнему определяются этим приближением. Выделенность кривых (11) проявляется при рассмотрении решений ОДУ, получаюшихся из (4) после линеаризации на фоне приближения бездисперсионного предела. Разъясним сказанное. После замен

$$
t=-\tau, \quad s=\frac{x}{\tau^{\frac{3}{2}}}, \quad b=\tau^{\frac{1}{2}} B, \quad a=\tau^{\frac{1}{2}} A
$$

система ОДУ (4) принимает следуюший сингулярно-возмушенный вид:

$$
\begin{aligned}
\tau^{-4}\left(2 B_{s s} B-B_{s}^{2}\right) & =3 B^{2}\left(A^{2}-B^{2}\right)-4 \frac{B^{2}}{\beta}+2 \gamma \tau^{-2}, \\
\tau^{-4}\left(A_{s s} B+3 A_{s} B_{s}+3 A B_{s s}\right) & =B\left(A^{3}-4 \frac{A}{\beta}-6 A B^{2}-2 \frac{s}{\beta}\right) .
\end{aligned}
$$

Предел (5) определяет главный член $\left(A_{0}(s), B_{0}(s)\right)$ решения системы $(13)$ :

$$
B_{0}^{2}=A_{0}^{2}-\frac{4}{3 \beta}, \quad \frac{5 \beta A_{0}^{3}}{2}-2 A_{0}+s=0 .
$$

Легко видеть, что сушествует формальное решение системы (13) вида

$$
A=A_{0}(s)+\sum_{0}^{\infty} \tau^{-2 k} A_{k}(s), \quad B=B_{0}(s)+\sum_{0}^{\infty} \tau^{-2 k} B_{k}(s)
$$

где $A_{k}$ и $B_{k}$ находятся из последовательности уравнений $(k=1,2, \ldots)$

$$
B_{0} B_{k}-A_{0} A_{k}=g_{k}(s), \quad 4 A_{0} B_{k}+B_{0} A_{k}=h_{k}(s),
$$


правые части которых зависят от функций $A_{n}(s), B_{n}(s)$ с $n<k$. Соответствующее формальное решение уравнений $(1),(2)$ при этом выглядит как

$$
\begin{aligned}
P(t, x)= & \tau^{\frac{1}{2}}\left[B_{0}(s)+O\left(\tau^{-2}\right)\right] \times \\
& \times \exp \left\{i\left[\tau^{2}\left(A_{0}\left(s-A_{0}+\frac{5 \beta A_{0}^{3}}{8}\right)+\frac{4}{3 \beta}\right)+\beta \gamma \ln \left(\tau B_{0}^{2}\right)+\Phi_{0}\right]\right\} .
\end{aligned}
$$

Эти формальные решения легко продолжаются и через прямую $t=0$. Функции $A_{k}(s), B_{k}(s)$ остаются при этом гладкими вплоть до кривых (6), на которых вместе с ростом номера $k$ нарастают и сингулярности $A_{k}(s), B_{k}(s)$.

В окрестности любой кривой $s=$ const сушествуют и истинные решения системы (13), которые в качестве полных асимптотических разложений имеют ряды (15). То же самое справедливо и при $t>0$ для всех кривых $s=$ const, расположенных ниже кривых "провала амплитуды" (6). Однако утверждение о сушествовании решения (13), которое для всех таких $s$ имеет асимптотические разложения (15), является неверным.

Дело в том, что обшее решение линеаризованной на фоне решения (15) системы ОДУ (13) претерпевает качественное изменение как раз при переходе через кривые (11). Это становится понятным при рассмотрении решений $A_{p}(s), B_{p}(s)$ следующей укороченной линеаризованной системы:

$$
\begin{aligned}
& \tau^{-4}\left(B_{p}\right)_{s s}=-3 B_{0}^{2} B_{p}+3 A_{0} B_{0} A_{p} \\
& \tau^{-4}\left(A_{p}\right)_{s s}=-\left(3 B_{0}^{2}+9 A_{0}^{2}\right) A_{p}-3 A_{0} B_{0} B_{p}
\end{aligned}
$$

У системы (17) существуют решения Венцеля-Крамерса-Бриллюэна (ВКБ) вида [5]

$$
A_{p}(s) \approx a_{p}(s) \exp \left(i \tau^{2} G(s)+\cdots\right), \quad B_{p}(s) \approx b_{p}(s) \exp \left(i \tau^{2} G(s)+\cdots\right) .
$$

При этом квадраты $k^{2}(s)$ производных $k(s)=G_{s}(s)$ имеют вид

$$
2 k^{2}=15 A_{0}^{2}-\frac{8}{\beta}+A_{0} \sqrt{3\left(15 A_{0}^{2}+\frac{16}{\beta}\right)} .
$$

Подстановка $P=b \exp (i S)$ сводит уравнение (1) к системе эволюционных уравнений

$$
S_{t}=2 b^{2}-a^{2}+b_{s s} b^{-1}, \quad b_{t}=2 a b_{s}+a_{s} b .
$$

После замен (12) из второго уравнения системы (19) следует, что

$$
\tau^{2} G=\frac{1}{12} \tau^{2} k\left(-\frac{15 \beta A_{0}^{3}}{8}+\frac{7 A_{0}}{4}-\sqrt{3\left(15 A_{0}^{2}+\frac{16}{\beta}\right)}\right) .
$$

Две ветви корня $\sqrt{3\left(15 A_{0}^{2}+16 / \beta\right)}$ определяют разные значения производной фазы $\tau^{2} k(s)$ и самой фазы $\tau^{2} G(s)$. Поэтому при переходе через кривые (11), на которых эти корни равны нулю, и происходит резкая смена в характере поведения ВКБ-решений (18): 
1) ниже кривых (11) мнимая часть фазы $\operatorname{Im}\left(\tau^{2} G(s)\right)$ всюду отлична от нуля. Соответствуюшие ВКБ-решения (18) при $\tau \gg 1$ экспоненшиально малы в случае $\operatorname{Im} G(s)>0$ и экспоненциально велики при $\operatorname{Im} G(s)<0$ (в рассматриваемой ситуации последний случай реализоваться не может);

2 ) выше же этих кривых функции $k(s)$ и $G(s)$ чисто вещественны. Это обстоятельство и приводит к изменению характера поправок к главному члену асимптотики $P(t, x)$, определяемому из уравнений (14).

Отметим, что задача об однозначном определении решений (1), (2) по их асимптотикам, определяемым из уравнений (14), “неустойчива", если речь идет об асимптотиках вдоль направлений, расположенных ниже кривых (11). Для остальных же направлений эта задача “устойчива". Рассматриваемые решения (1), (2) описывают сглаживающее влияние малой дисперсии на зарождение процессов самопроизвольного падения интенсивности приближения нелинейной геометрической оптики (НГО) [6-9]. Следовательно, в некоторых окрестностях точек зарождения этих процессов имеются две направленные "вниз" кривые, лиш “ниже” которых задача восстановления решений полных уравнений с малой дисперсией по их приближениям НГО “неустойчива". Таким образом, в данной статье описывается контрпример к известному общему "принципу хрупкости хорошего" [10].

4. Опишем переходную асимптотику $P(t, x)$ в окрестностях кривых (11). Для определенности возьмем случай правой кривой

$$
s=s_{\text {п }}=\frac{14}{3} \sqrt{-\frac{16}{15 \beta}} .
$$

Случай левой кривой рассматривается совершенно аналогично. При $s \rightarrow s_{\text {п часть }}$ функций $i G(s)$ разлагается в ряды

$$
i G(s)=-i 6 \sqrt{-\frac{5}{\beta}}+i 2 \sqrt{-\frac{3}{\beta}}\left(s-s_{\Pi}\right)-i 25^{-\frac{3}{4}} 3^{-\frac{5}{4}}(-\beta)^{-\frac{1}{4}}\left(s-s_{\Pi}\right)^{\frac{3}{2}}+\cdots,
$$

а остальные являются их комплексными сопряжениями. При $s<s_{\text {п }}$ рассматриваются лишь $i G$ с неположительной вешественной частью.

Разложения (20) в соответствии с методом согласования [11] определяют следуюший выбор "медленной":

$$
y=\tau^{\frac{4}{3}}\left(s-s_{\Pi}\right),
$$

и “быстрой":

$$
H=-\tau^{2} 6 \sqrt{-\frac{5}{\beta}}+\tau^{\frac{2}{3}} 2 \sqrt{-\frac{3}{\beta}} y,
$$

переменных. При таком выборе для $|y|=O(1)$ "быстрая" переменная $H\left(\tau^{2}, y\right)$ в главном порядке "сшивается" с соответствуюшими фазами $\tau^{2} G(s): i \tau^{2} G(s)-H\left(\tau^{2}, y\right)=O(1)$. После замены (21) система (13) принимает вид

$$
\begin{gathered}
\tau^{-\frac{4}{3}}\left(2 B_{y y} B-B_{y} 2\right)=3 B^{2}\left(A^{2}-B^{2}\right)-4 \frac{B^{2}}{\beta}+2 \gamma \tau^{-2} \\
\tau^{-\frac{4}{3}}\left(2 A_{y y} B+3 A_{y} B_{y}+3 A B_{y y}\right)=B\left(A^{3}-4 \frac{A}{\beta}-6 A B^{2}-2 \frac{s_{\Pi}}{\beta}\right)-2 \tau^{-\frac{4}{3}} \frac{y B}{\beta} .
\end{gathered}
$$

4 Теоретическая и математическая физика, т. 118, № 3, 1999 г. 
Решение (23) ищется в виде рядов по степеням малого параметра $\tau^{-\frac{2}{3}}$ :

$$
\begin{aligned}
A & =A^{0}+\tau^{-\frac{2}{3}} A^{1}+\tau^{-\frac{4}{3}} A^{2}+\cdots, \quad B=B^{0}+\tau^{-\frac{2}{3}} B^{1}+\tau^{-\frac{4}{3}} B^{2}+\cdots, \\
A^{0} & =A_{0}\left(s_{\Pi}\right)=\sqrt{-\frac{16}{15 \beta}}, \quad B^{0}=B_{0}\left(s_{\Pi}\right)=\sqrt{-\frac{12}{5 \beta}} \\
A^{n} & =A^{n}(y, H), \quad B^{n}=B^{n}(y, H) .
\end{aligned}
$$

Введение переменной (22) "регуляризует" систему (23). И в результате подстановки в нее рядов (24) возникает последовательность систем ОДУ по $H$ :

$$
\begin{aligned}
B_{H H}^{1}+3 B_{0}^{2}\left(s_{\Pi}\right) B^{1}-3 A_{0}\left(s_{\Pi}\right) B_{0}\left(s_{\Pi}\right) A^{1} & =0, \\
A_{H H}^{1}-\left(3 B_{0}^{2}\left(s_{\Pi}\right)+9 A_{0}^{2}\left(s_{\Pi}\right)\right) B^{1}+3 A_{0}\left(s_{\Pi}\right) B_{0}\left(s_{\Pi}\right) B^{1} & =0 ; \\
B_{H H}^{n}+3 B_{0}^{2}\left(s_{\Pi}\right) B^{n}-3 A_{0}\left(s_{\Pi}\right) B_{0}\left(s_{\Pi}\right) A^{n} & =R_{n}(y), \\
A_{H H}^{n}-\left(3 B_{0}^{2}\left(s_{\Pi}\right)+9 A_{0}^{2}\left(s_{\Pi}\right)\right) B^{n}+3 A_{0}\left(s_{\Pi}\right) B_{0}\left(s_{\Pi}\right) B^{n} & =Q_{n}(y) \quad(n>1) .
\end{aligned}
$$

Правые части уравнений (25) зависят лишь от функциий $A^{1}, B^{1}, A^{2}, B^{2}, \ldots, A^{n-1}$, $B^{n-1}$. Из “сшивки" с (18) и вешественности $B(\tau, s), A(\tau, s)$ следует, что

$$
B^{1}=u(y) \exp (i H)+u^{*}(y) \exp (-i H), \quad A^{1}=-u(y) \exp (i H)-u^{*}(y) \exp (-i H) .
$$

Налагая обычное требование ограниченности решений цепочки (25) по $H$, мы последовательно находим все члены разложения (24), по пути определяя не зависящие от $H$ функции, которые возникали на предыдущих шагах. В частности, из требования ограниченности по $H$ решений систем (25) при $n=2$ и $n=3$ получаем, что $u(y)$ в (26) должно быть решением ОДУ:

$$
u_{y y}=-\frac{y u}{5 \sqrt{-15 \beta}}-\frac{8|u|^{2} u}{5} .
$$

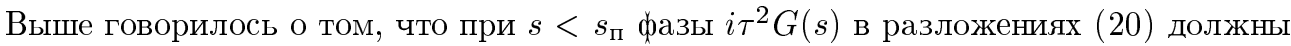
иметь отрицательную вещественную часть. Отсюда вытекает требование экспоненциальной малости решений ОДУ (27). Поэтому эти решения сводятся к вешественным решениям (8) при помоши замен $(\phi=$ const $)$

$$
y=-5^{-\frac{1}{2}}(-3 \beta)^{-\frac{1}{6}} z, \quad u(y)=5^{\frac{1}{2}}(-3 \beta)^{\frac{1}{6}} \exp (i \phi) v(z) .
$$

5. Все вешественные решения уравнения Пенлеве (8) являются гладкими и имеют [12] следуюшую асимптотику при $z \rightarrow-\infty$ :

$$
v(z)=c(-z)^{-\frac{1}{4}} \sin \left(\frac{2}{3}(-z)^{\frac{3}{2}}+\frac{3}{4} c^{2} \ln (-z)+\chi\right) .
$$

В нашем случае $v(z)$ также должно экспоненциально убывать при $z \ll 1$. Такие решения сушествуют и имеют при $z \rightarrow \infty$ асимптотику [12]

$$
v(z)=\frac{a}{2 \sqrt{\pi}} z^{-\frac{1}{4}} \exp \left(-\frac{2}{3} z^{\frac{3}{2}}\right) .
$$


При этом имеют место следуюшие формулы связи $(l=0,1)$ :

$\chi=\frac{3}{2} c^{2} \ln 2-\frac{\pi}{4}-\arg \Gamma\left(i \frac{c^{2}}{2}\right)+l \pi(\bmod (2 \pi)), \quad a^{2}=\exp \left\{\pi c^{2}\right\}-1, \quad \operatorname{sign} a=1-2 l$. Формулы (28), (29) согласуются с разложениями (20) фаз $\tau^{2} G(s)$ ВКБ-решений (18).

Напомним, что ниже кривых (11) знак мнимой части $G(s)$ всюду отрицателен. Этот факт гарантирует то, что каждое решение (1), (2), имеюшее разложение (16) вдоль любой кривой, расположенной ниже кривых (11), будет иметь асимптотику (16) и всюду в области $J$, расположенной ниже этих кривых. Однако он же, как уже отмечалось, означает, что справедливость в области $J$ асимптотики (16) еше не определяет однозначно соответствуюшее решение $P(t, x)$.

6. Решения НУШ (1), удовлетворяюшие уравнению (2), являются изомонодромными [13]. Наряду с уравнениями [14] представлений нулевой кривизны для уравнения (1)

$$
\Psi_{x} \Psi^{-1}=L(x, t, \lambda), \quad \Psi_{t} \Psi^{-1}=Q(x, t, \lambda)
$$

соответствуюшие $\Psi$-функции являются одновременно решениями ОДУ по $\lambda$, т. е.

$$
\begin{aligned}
\Psi_{\lambda} \Psi^{-1} & =\left[-i\left(4 \beta \lambda^{3}+4 \lambda t-2 \beta|P|^{2} \lambda+x\right)-\beta\left(P_{x} P^{*}-P_{x}^{*} P\right)\right]\left(\begin{array}{cc}
1 & 0 \\
0 & -1
\end{array}\right)+ \\
& +\left(\begin{array}{cc}
0 & 4 i \beta \lambda^{2} P-2 \beta \lambda P_{x}+4 i t P-\beta P_{t} \\
4 i \beta \lambda^{2} P^{*}+2 \beta \lambda P_{x}^{*}+4 i t P^{*}+\beta P_{t}^{*} & 0
\end{array}\right) .
\end{aligned}
$$

Для секторов $S_{j}=\{\lambda: \pi(j-1) / 4<\arg \lambda<\pi(j+1) / 4, j=1,8\}$ сушествуют [15] решения $\Psi_{j}(31)$, которые в этих секторах обладают асимптотикой

$$
\Psi_{j} \approx \exp \left[-i\left(\lambda x+2 t \lambda^{2}+\beta \lambda^{4}-\beta \gamma \ln \lambda\right)\right]\left(\begin{array}{cc}
1 & 0 \\
0 & -1
\end{array}\right) .
$$

Из-за того что $\Psi_{j}$ удовлетворяют ОДУ $(30)$, матрицы Стокса $M_{j}=\Psi_{j}{ }^{-1} \Psi_{j+1}$ не зависят от $t$ и $x$. При этом они имеют вид

$$
M_{2 k}=\left(\begin{array}{cc}
1 & m_{2 k+1} \\
0 & 1
\end{array}\right), \quad M_{2 k+1}=\left(\begin{array}{cc}
1 & 0 \\
m_{2 k} & 1
\end{array}\right) .
$$

Набор множителей Стокса $m_{j}$ (составляюших в рассматриваемой ситуации полньй набор так называемых данных монодромии) находится во взаимно однозначном соответствии с множеством совместных решений уравнений (1) и (2). К сожалению, априори очевидной информацией об асимптотике интересующего нас изомонодромного решения уравнений (1), (2) мы обладаем лишь в области $J$, расположенной ниже кривых (11). В области же $J$, как отмечалось в конце п. 5 , эта асимптотика не определяет соответствующих решений $P(t, x)$ однозначно. Однако часть данных монодромии из этой асимптотики все-таки находится. Для всех совместных решений уравнений (1), (2), обладающих в $G$ асимптотикой (16), их множители Стокса $m_{2}, m_{3}, m_{6}, m_{7}$ имеют вид

$$
\begin{array}{ll}
m_{2}=\exp \left(i \Phi_{0}+i \beta \gamma \ln 4-\pi \beta \gamma\right), & m_{3}=-\exp \left(-i \Phi_{0}-i \beta \gamma \ln 4+\pi \beta \gamma\right), \\
m_{6}=-\exp \left(i \Phi_{0}+i \beta \gamma \ln 4+\pi \beta \gamma\right), & m_{7}=\exp \left(-i \Phi_{0}-i \beta \gamma \ln 4-\pi \beta \gamma\right) .
\end{array}
$$


Кроме того, в этом случае справедливы соотношения

$$
m_{1} m_{2}+m_{3} m_{4}=-1, \quad m_{5} m_{6}+m_{7} m_{8}=-1 .
$$

Этой информации оказывается достаточно для того, чтобы сделать вывод об обязательности малоамплитудных осциллирующих добавок к асимптотике (16) после перехода по крайней мере через одну из кривых (11).

7. Среди решений второго уравнения Пенлеве (8), обладаюших асимптотиками (28), $(29)$, имеется и тривиальное решение $v(z)=0$. В этом случае, очевидно, при переходе через правую из кривых (11) никакой перестройки в характере рассматриваемого решения не происходит. Асимптотика (16) остается справедливой вплоть до оси $t=0$, а после небольшого изменения без затруднений продолжается и до правой из кривых (6). Этой ситуации отвечает случай множителя Стокса $m_{1}=0$, в чем нетрудно убедиться непосредственным вычислением, например, при $t=0$ и $x \rightarrow \infty$. Аналогичным образом предположение о сохранении справедливости асимптотики (16) при переходе через левую из кривых (11) влечет за собой вывод о том, что в этом случае $m_{4}=0$. Одновременное же равенство нулю $m_{1}$ и $m_{4}$ противоречит первому из соотношений (32).

В ложности предположения о сохранении асимптотики (16) при переходе как через правую, так и через левую кривые (11) можно убедиться и без непосредственного вычисления данных монодромии. Допустим, например, что асимптотика (16) сохраняется при переходе через правую из этих кривых. Легко видеть, что тогда гладкий переход через кривую (6) описьвается соотношением (9), в котором $r=0$, а решение уравнения (10), как и в случае аналога интеграла Пирси из [2], лишь постоянным множителем отличается от вещественного решения второго уравнения Пенлеве (8). Только в данном случае это решение $v(z)$ при $z \rightarrow \infty$ не убывает экспоненциально, а разлагается в асимптотический ряд $v(z)=(z / 2)^{\frac{1}{2}}+8 \sqrt{2} z^{-\frac{5}{2}}+\cdots$. При $z \rightarrow-\infty$ оно имеет асимптотику (28), параметры $c$ и $\chi$ которой определяются из результатов работы [16]. Из формулы (28) следует, что внутри кривых (6) соответствуюшее совместное решение уравнений (1), (2) имеет двухфазовые высокочастотные колебания амплитуды порядка $O\left(t^{-\frac{1}{2}}\right)$, определяемые двумя корнями кубического уравнения $(7)$, которые на правой из кривых (6) совпадают и имеют на ней особенность типа квадратного корня.

Однако на левой кривой (6) эти корни отличны, и один из них при этом не имеет особенностей. Стало быть, в таком случае в асимптотике (9), описывающей гладкий переход в окрестности кривой (6), постоянная $r$ неизбежно отлична от нуля. А значит, ниже этой кривой к главному члену асимптотики (16) обязательно имеется поправка амплитуды порядка $O\left(t^{-\frac{1}{2}}\right)$, представляюшая собой высокочастотные осцилляции. Из сказанного в п. 3 следует, что эти осцилляции имеют место вплоть до кривых (11).

Эти колебания являются двухфазовыми. Их двухфазовый характер следует из требования согласованности с приведенной в п. 4 структурой асимптотики, описывающей исчезновение высокочастотных малоамплитудных поправок к (15) при $t \rightarrow-\infty$ в процессе перехода через левую из кривых (11) во внутренность области $J$.

Поправки порядка $O\left(t^{-2}\right)$ к решению (16), представляющие собой двухфазовые высокочастотные колебания, имеют место в обеих областях между кривыми (6) и (11) тог- 
да, когда отличны от нуля оба решения (8), определяющие асимптотики совместных решений уравнений $(1),(2)$ в окрестностях кривых (11).

8. После перехода из области $J$ через кривые (11) такие колебания обязательно возникнут у всех четных совместных решений уравнений $(1),(2)$, имеющих в области $J$ асимтотику (16). При этом условие справедливости в области $J$ решения (16) совместимо для четных функций $P(t, x)$ с условием стремления этих функций к нулю при $t \rightarrow \infty$ внутри областей, ограниченных кривыми (6).

Действительно, при $x=0$ четное решение уравнений $(1),(2)$ удовлетворяет ОДУ

$$
P_{t t}=\left(\frac{4 i t}{\beta}+2 i|P|^{2}\right) P_{t}+\left(2 i+8 t|P|^{2}\right) \frac{P}{\beta} .
$$

Заметим, что уравнение (33) легко сводится [17] к четвертому уравнению Пенлеве.

Изучение асимптотик при $t \rightarrow \pm \infty$ всевозможных решений (33) было проведено в работах $[18,19]$ при помоши метода изомонодромных деформаций. Из этих работ следует, что среди решений уравнения (33), обладающих асимтотикой (16) при $t \rightarrow-\infty$, имеется целое семейство (непрерьвно зависяшее от еше одного дополнительного параметра), которое убывает при $t \rightarrow \infty$. Главный член асимптотики при этом задается выражениem

$$
P(t, x)=t^{-\frac{1}{2}}\left[d \exp \left(-i\left(\beta \gamma+4|d|^{2}\right) \ln t\right)+e \exp \left(\frac{2 i t^{2}}{\beta}+i\left(\beta \gamma-2|e|^{2}\right) \ln t\right)\right],
$$

где комплексные константы $d$ и $е$ связаны соотношением $|d|^{2}=|e|^{2}-\beta \gamma / 2$. Используя решение (34) в качестве начальных данных для ОДУ (2), нетрудно выписать и асимптотики $P(t, x)$ внутри кривых (6). Мы их здесь не приводим. Отметим только, что качественно они подобны асимптотике интеграла Пирси (и его аналога из работы [2]).

9. Однако из работ $[18,19]$ следует также, что асимптотики (16) и (34) совместимы отнюдь не для всех четных решений (1), (2). Кроме того, из изложенного в п. 8 следует, что справедливость асимптотик (16) внутри области $J$ совместима с требованием стремления к нулю при $t \rightarrow \infty$ не только для четных решений $P(t, x)$ (вопреки ошибочному утверждению работы [1]).

K сожалению, из-за большого объема необходимых аналитических выкладок, нам пока не удалось описать полностью класс совместных решений уравнений (1), (2), которые при $x^{2}+t^{2} \rightarrow \infty$ стремятся к нулю внутри области, ограниченной кривыми $(6)$, и определяются приближением (14) вне ее. Тем не менее использование метода согласования [11] и результатов данной статьи позволяет сделать заключение о наличии следуюшего эффекта (не обнаруженного в предыдуших работах): по соседству ( по крайней мере, с одной стороны) с типичной областью малоамплитудных высокочастотных колебаний, совпадающей с областью нулевой амплитуды приближения НГО, развиваются также высокочастотные колебания с амплитудой порядка $O\left(\epsilon^{\frac{1}{2}}\right)$. Однако они происходят уже на фоне приближения НГО ненулевой амплитуды.

Благодарности. Авторы благодарят за поддержку Российский фонд фундаментальных исследований (грант 96-01-00382). Б.И. Сулейманов благодарен также Фонду поддержки ведущих научных школ России (проект 96-15-96241). 


\section{Список литературы}

[1] В.Р. Кудашев, Б. И. Сулейманов. Письма в ЖЭТФ. 1998. Т. 62. Вып.4. С. 358.

[2] R. Haberman, R. Sun. Stud. Appl. Math. 1985. V. LXXII. № 1. P. 39.

[3] Б. И. Сулейманов. Зап. научн. семин. ЛОМИ. 1991. Т. 187. С. 110.

[4] A.A. Kanaeв. ТМФ. 1988. Т. 77. № 3. С. 323.

[5] М. В. Федорюк. Асимптотические методы для линейных обыкновенных дифференциальных уравнений. М.: Наука, 1983.

[6] А. Б. Шварибург. Геометрическая оптика в нелинейной теории волн. М.: Наука, 1976.

[7] А. В. Гуревич, А. Б. Шварибург. ЖЭТФ. 1970. Т. 58. Вып.6. С. 2012.

[8] С.К. Жданов, Б. А. Трубников. Квазигазовые неустойчивые среды. М.: Наука, 1991.

[9] К. А. Наугольных, Л. А. Островский. Нелинейные волновые процессы в акустике. М.: Наука, 1990.

[10] В. И. Арнольд. Теория катастроф. М.: Наука, 1990.

[11] A. М. Ильин. Согласование асимптотических разложений решений краевых задач. М.: Наука, 1989

[12] A. Р. Иmс, А. А. Капаев. Изв. АН СССР. Сер. матем. 1987. Т. 51. № 4. С. 878.

[13] А. Р. Итс. Изв. АН СССР. Сер. матем. 1985. Т. 49. № 3. С. 530.

[14] В. Е. Захаров, А. Б. Шабат. ЖЭТФ. 1972. Т. 61. № 1. С. 118.

[15] В. Вазов. Асимптотические разложения решений обыкновенных дифференциальных уравнений. М.: Мир, 1968.

[16] А. Н. Белогрудов. Дифф. уравнения. 1997. Т. 33. № 5. С. 587.

[17] A. V. Kitaev. J. Math. Phys. 1994. V. 35. № 6. P. 2934.

[18] А. В. Китаев. Зап. научн. семин. ЛОМИ. 1988. Т. 169. С. 84.

[19] A. A. Капаев. Трансценденты Пенлеве как нелинейные специальные функции. Дисс. на соискание уч. ст. докт. физ.-мат. наук. Санкт-Петербург: Санкт-Петербургское отделение Математического института им. В. А. Стеклова РАН, 1998. 